\title{
An optoelectronic detecting based environment perception experiment for primer students using multiple- layer laser scanner
}

Shifeng Wang, Rui Wang, Pengfei Zhang, Xiang Dai, Dawei Gong

Shifeng Wang, Rui Wang, Pengfei Zhang, Xiang Dai, Dawei Gong, "An optoelectronic detecting based environment perception experiment for primer students using multiple-layer laser scanner," Proc. SPIE 10452, 14th Conference on Education and Training in Optics and Photonics: ETOP 2017, 104523F (16 August 2017); doi: 10.1117/12.2270973

Event: 14th Conference on Education and Training in Optics and Photonics, ETOP 2017, 2017, Hangzhou, China 


\title{
An opto-electronic detecting based environment perception experiment for primer students using multiple-layer laser scanner
}

\author{
Shifeng Wang, Rui Wang, Pengfei Zhang, Xiang Dai, Dawei Gong \\ National Demonstration Center for Experimental Opto-Electronic Engineering Education, School of \\ Opto-Electronic Engineering, Changchun University of Science and Technology, 7089 Weixing \\ Road, Changchun, China, 130022.
}

\begin{abstract}
One of the motivations of OptoBot Lab is to train primer students into qualified engineers or researchers. The series training programs have been designed by supervisors and implemented with tutoring for students to test and practice their knowledge from textbooks. An environment perception experiment using a 32-layer laser scanner is described in this paper. The training program design and laboratory operation is introduced. The four parts of the experiments which are preparation, sensor calibration, 3D space reconstruction, and object recognition, are the participating students' main tasks for different teams. This entire program is one of the series training programs that play significant role in establishing solid research skill foundation for opto-electronic students.
\end{abstract}

Keywords: Multiple-layer laser scanner, Environment perception, Undergraduate student, Open-Lab Program

\section{INTRODUCTION}

According to the national tertiary education guide [1], the education of undergraduate is encouraged to train the students obtain not only the specialist knowledge but also the practical abilities and innovation desirability. Particularly, the engineering students are encouraged to participate more contests or competitions to practice their knowledge learned from textbooks. Moreover, the undergraduate students with Optical Engineering specialty or Measurement and Control Technology and Instrument of our university are having more opportunities to join a laboratory to obtain solid research skill foundation for possible further education.

Three main specialties are all related to Opto-Electronic techniques, which belong to National Demonstration Center for Experimental Opto-Electronic Engineering Education, the school of Opto-Electronic Engineering school, Changchun University of Science and Technology (CUST), China. All the students in this school are divided into different levels or classes. The Open-Lab Program [2] allows the students who are willing to involve in more practical activities and enhance their abilities to solve engineering problems. Concerning the students' taught subjects received, an advantage optical sensor (a multiple-layer laser scanner) is selected to play the key role of the training activities. A series experiments are then designed and implemented in the OptoBot Laboratory under the supervising and instrument. Two cycles of this program have presented productive and impressive results.

\section{THE PROGRAM DESIGN AND OPTOBOT LABORATORY OPERATION}

\subsection{Current status of the undergraduate students}

There are approximately 2,000 undergraduate students registered in Opto-Electronic Engineering School. Around half of them take the specialist courses when they come to year three and year four. This is the most appropriate period to build up their professional skills, research interests, and potential various careers. In recent years, we found out that these students plan their future clearer and more realistic. Most of them have already made settle down goals before year three. Generally speaking, some of them plan to have further education pursuing master's degree or doctoral degree, domestically or abroad. Some of them plan to be employed by companies. Others even plan to start their own companies applying their professional skills. Majority of these students are longing to have more practical and more specific skills beyond the regular experimental courses. Some of them even ask for more freely labs and equipments to practice their thoughts and designs rather than following the experiment instructions step by step. The current regular experiment courses cannot satisfy the demands of these students.

14th Conference on Education and Training in Optics and Photonics: ETOP 2017, edited by Xu Liu,

Xi-Cheng Zhang, Proc. of SPIE Vol. 10452, 104523F · C 2017 ICO, IEEE, OSA, SPIE

CCC code: $0277-786 X / 17 / \$ 18 \cdot$ doi: $10.1117 / 12.2270973$ 


\subsection{The program design}

Under these circumstances, our OptoBot Lab made a series programs to train the students to achieve their purposes. We concerned few facts to design the programs. 1) An appropriate difficulty. The students cannot learn much if it is too easy whereas too hard makes the students loss their enthusiasm. 2) It should leave the possible growing space for the student. There must be enough innovation work to do for them. Both academic way and engineering way are possible. 3) It should have some advance in the field internationally, which guides them into possible research interests. These research interests could be the fundamental for their further education, either abroad or domestically.

\subsection{The related experimental platform}

One of the facilities of the OptoBot Lab is a wheeled robot, as shown in figure 1, which is employed for environment perception research work. The platform is equipped with a long-range single-layer laser scanner (SICK LMS151), a short-range single-layer laser scanner (Hokuyo UTM-30LX), two multiple-layer laser scanner (IBEO LUX8L and Velodyne HDL-32E), two cameras (AVT Pike F-100C), an embedded computer, and batteries. The platform can be used for data collection and control algorithm verification experiments. The platform can be remotely operated by a laptop via WIFI. As shown in figure 1, the Velodyne HDL-32E as a typical optical sensor among those sensors was decided to be the main device used for this open-lab program.

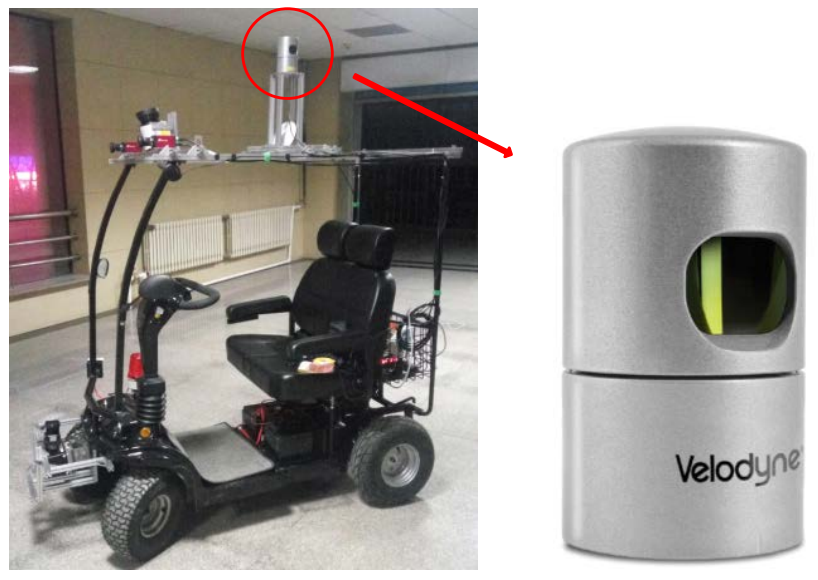

Figure 1 The multiple-layer laser scanner on the experimental platform

\subsection{The OptoBot Lab and the program operation}

An undergraduate student committee takes the charge of the daily management of the group. The students who join the program are divided into few teams. Each team takes a specific project to devote. A few graduate students perform as advisers. A teacher performs as supervisor of the whole lab. Regular presentations are held for sharing the new ideals and reporting the progresses. Workshops and showcases are also welcome which can spread the influence of the lab and bring more students to join in.

\subsection{The students selection and the program completion}

Normally, we accept all grades of the undergraduate students, but year one or year two ones are more welcome. Some of them are recommended by the current lab members. Others get to know the lab via posters or website. In the first round, we will select part of them from all the applications by an interview. Then some simple and basic tasks will be given to these selected students. After one month probationary period, a two-way choice will be made by both student and lab. Generally, the project using multiple-layer laser scanner is the most popular one. Afterwards, the formal program will be implemented for one year. The students who achieve reasonable and satisfactory results will receive the academic credits and reference letters. 


\section{THE MULTIPLE-LAYER LASER SCANNER EXPERIMENTS}

In this experiment, undergraduate students will be divided into three teams, which are team A, team B, and team C. All the students will do the preparation part of the experiments. Each team will choose a different experiment from the remaining three experiments.

\subsection{Preparation of the experiments}

A typical multiple-layer laser scanner is employed in the experiments which is a Velodyne product HDL-32E [3]. The basic operation of the laser sensor will be explained to the members of the three teams. Next step is data acquisition. Basically, there are two ways for date logging. 1) One is to use the Windows PC software provided by the manufacturer. 2) The other is based on the Robot Operating System (ROS) [4], which is a collection of tools, libraries, and conventions that aim to simplify the task of creating complex and robust robot behavior across a wide variety of robotic platforms. Comparing above the two ways, the operation of the former one is much easier than the last one. However, considering the second way could obtain multiple data from different sensors at the same time, the second one is selected. Then we will show the students how to install the ROS in their computer and teach them the basic commands. The Matlab is chosen for the logged data processing.

\subsection{Calibration experiment}

There are some errors in the measurement data of the HDL-32E and therefore the calibration experiment is of great significance for the follow-up work [5]. The students of team A are required to analysis the errors in the following three parts: measuring distance, horizontal angle resolution, vertical angle resolution. For each part, the gross error and systematic error analysis should be involved. Besides, the errors would be also influenced by the different background light and incident angle. The errors between the different devices, which are the same model, are supposed to take into account.

\subsection{D space reconstruction experiment}

The logged data from the HDL-32E is point cloud data frame by frame. The students of team B are asked to find out the corresponding points from two different frames in a moving platform to reconstruct the 3D space observed [6]. Then register the frames by the corresponding points or features. It is a committed step in the experiment. The Point Cloud Library (PCL) [7] is an important and remarkable tool for the 3D space reconstruction task. Combining the PCL with the ROS, the accuracy of the 3D space reconstruction will be effectively improved.

\subsection{Objects recognition experiment}

Once the data of point cloud scanned by HDL-32E is obtained, clustering and feature extraction from the observed objects are implemented with some related algorithm by team $\mathrm{C}$ [8]. The purpose of clustering is to separate the point cloud points into object by object. The feature selection is then implemented for each object for the following machine learning process. Then the classification of objects using this multiple-layer laser is expected to be done.

\section{THE PROGRAM EFFECT}

The program has implemented for three rounds. The remarkable effect has happened. The involved undergraduate students have been greatly improved their engineering skill. The abilities of carrying out research activity have also been trained. Most of them have been brought into house of science though this program. The application of the multiple-layer laser scanner could be used again in their future education or research in one way or another. The higher possibility to obtain further education also can be expected. Moreover, their abilities of teamwork and the researcher community are also enhanced and expanded.

\section{ACKNOWLEDGEMENTS}

This work is funded by National Natural Science Foundation of Jilin Province (20150101047JC), Changchun University of Science and Technology (CUST) for Young Scholar (XQNJJ-2014-05), project of reform for the computer assistant design of instrument component subject (“《仪器零件》上机实验教学改革的研究与实践”教改项目). This work is also supported by OptoBot Lab, National Demonstration Center for Experimental Opto-Electronic Engineering Education, School of Opto-Electronic Engineering, School of Opto-Electronic Engineering, CUST. 


\section{REFERENCES}

[1] “National Long-Term Education Reform and Developing Outline (2010-2020)", http://www.gov.cn/jrzg/201007/29/content_1667143.htm

[2] "The 2017 Innovation Open-Lab Program for the Undergraduate Students of the School of Opto-Electronic Engineering, Changchun University of Science and Technology”, http://gd.cust.edu.cn/xytz/25814.htm

[3] "Velodyne HDL-32E data sheet”, http://velodynelidar.com/hdl-32e.html

[4] “About ROS”, http://www.ros.org/about-ros/

[5] Chan, T. O., Lichiti, D. D., Belton, D. "Temporal analysis and automatic calibration of the Velodyne HDL-32E LiDAR system”, ISPRS Annals of Photogrammetry, Remote Sensing and Spatial Information Sciences, Volukme II-5/W2, 61-66(2013).

[6] Hammoudi, K. "Contributions to the 3D city modeling: 3D polyhedral building model reconstruction form aerial images and 3D façade modeling from terrestrial 3D point cloud and image”, Ph.D. thesis in signal and image processing, Université Paris-Est, 234p., 2011.

[7] “About PCL”, http://pointclouds.org/about/

Hragh, M., Jørgensen, R., Pedersen, H. "Object detection and terrain classification in agricultural fields using 3D lidar data”, International Conference on Computer Vision Systems, ICVS2015: Computer Vision Systems, 188-197(2015). 